\title{
The Electrodynamic Human Motion Energy Converter with Planar Structure
}

\author{
J.Blums $^{1, a}$, G.Terlecka ${ }^{2, b}$, A.Vilumsone ${ }^{2, c}$ \\ ${ }^{1}$ Institute of Technical Physics, \\ Riga Technical University, 14/24-322, Azenes str., Riga, LV 1048, Latvia \\ ${ }^{2}$ Institute of Textile Materials Technologies and Design, \\ Riga Technical University, 14/24-319, Azenes str., Riga, LV 1048, Latvia \\ ablum@latnet.Iv, bgalina.terlecka@rtu.Iv, causma.vilumsone@rtu.lv
}

Keywords: energy harvesting, electrodynamic generator, wearable energy sources.

\begin{abstract}
The performance of the electrodynamic human motion energy converter with planar structure is under investigation. The electrodynamic converter consists of a flat, spiral-shaped coil and a rectangular magnet that during human motion moves in respect to one another. During the motion of the rectangular magnet with a remanence $0.1 \mathrm{~T}$ over a flat, formed up as an Archimedes spiral coil with 25 turns, an average power of about $10 \mu \mathrm{W}$ is generated. The generator elements can both be deployed on a variety of clothing items which move relative to one another during the human motion, and also realizing the forced oscillation of the magnet during walking.
\end{abstract}

\section{Introduction}

Electronics get smaller in size, offer ample opportunities to integrate into human apparel and accessories, giving them additional features (security, communication, entertainment, health monitoring, etc.) [1]. To operate such devices nowadays mainly rechargeable accumulators or batteries are used, which imposes an obligation on the wearer to replace or recharge them periodically. The reduction of power consumed by such devices induces a growing interest in devices suitable for generation of microwatt power from the human motions over the last few years $[2,3]$. Moving (walking, running) person make a lot of equal, periodic motions, which can be used as a mechanical energy source for electricity generation. In recent years, research is mainly carried out in three directions: the application of the piezoelectric effect, the use of electrostatic forces and electromagnetic induction or the electrodynamic method [3]. The first two methods are found to be effective in energy harvesting for industrial oscillations (frequency range 1-10 kHz), but the electrodynamic method - for a human movement frequency range $(0.1$ to $100 \mathrm{~Hz})$.

The apparel integrated electronic devices should be made as to be utilized easily, they should not change the size and weight of the clothing essentially and should not lose operation after a laundry care process. It is also important not to create thick connections, significantly alter the visual properties of textile structures, which are defined by the thread structure, mobility grade and flexibility, ensuring comfortable movement, like ordinary apparel. Proposed electromagnetic (EM) transducers, which convert mechanical energy of human motions into electrical energy, are created as three-dimensional devices with a conventional cylindrical coil, with a magnetic element moving inside [3]. This construction is not suitable to be integrated into clothing since each item of the apparel consists of two-dimensional parts. The aim of this study is to explore the possibilities of creating an EM generator with a flat architecture and to evaluate its developed power.

\section{Experimental}

Generator design. In this study, the flat coil (FC) of an EM converter is formed in one plane, as a spiral with regularly increasing radius of curvature (Archimedes spiral, Fig.1.) in a way that the windings do not touch each other. It provides isolation to the windings, prevents unwanted electrical contact between adjacent turns due to the motion and deformation of the parts of clothing. A blockshaped permanent magnet, moving parallel to the plane of the FC, without crossing it, creates an alternating magnetic field flux, which is required for the induction of an electromotive force. 
Simultaneously with the FC geometry the generator's minimal volume and occupied space is achieved and it is suitable to be fully integrated into clothing. Key components of the investigated electrodynamic converter are shown in Fig.1: 1 - the FC, 2 - powered device or the system of energy conversion and storage and 3 - the trajectory of the motion of the block-shaped magnet. The magnet has to move in such a way that the trajectory of the poles motion coincides with line MM'.

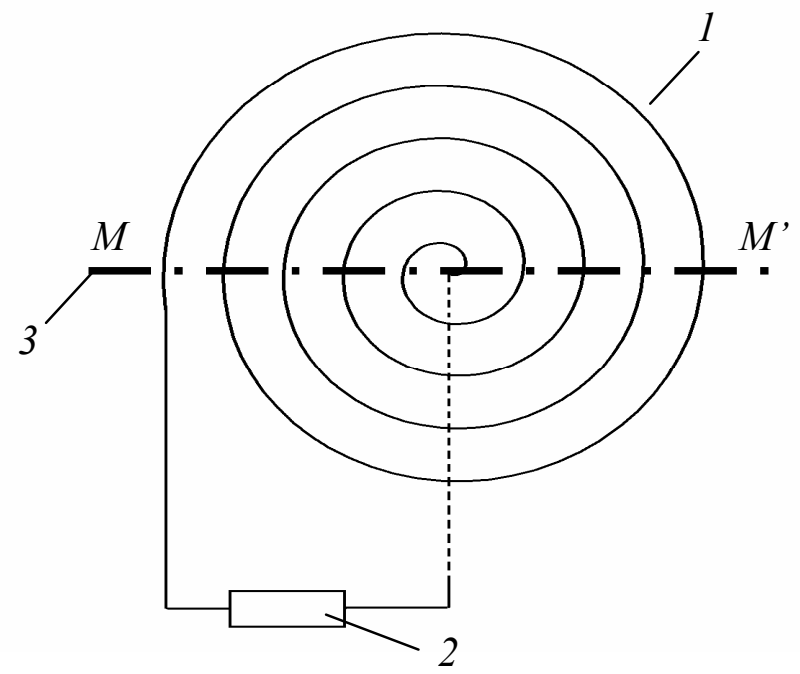

Fig. 1. Electrodynamic converter: 1 coil; 2 - powered device or energy accumulation system; 3 - the path of magnet oscillations.

Manually created coils with the number of turns from 5 to 75 were used for the research. The coil wire (copper wire with a diameter of 0.22 and $0.1 \mathrm{~mm}$ ) was formed on a flexible and insulating substrates (cloth, adhesive tape). A variable magnetic field was created by permanent block-shaped magnets with linear dimensions $8 \times 5 \times 3 \mathrm{~mm}$ and a remanence of $0.37 \mathrm{~T}$ and $20 \times 10 \times 5 \mathrm{~mm}$ with a remanence of $0.1 \mathrm{~T}$. The power of the generator was registered in two modes: induced electromotive force (as no-load voltage) and maximum power (using the load with a resistance, which is equal to the coil resistance) mode. The oscillation frequency of the magnets was in the range from $3-5 \mathrm{~Hz}$, corresponding to a human arm movement frequency of fast walking and running.

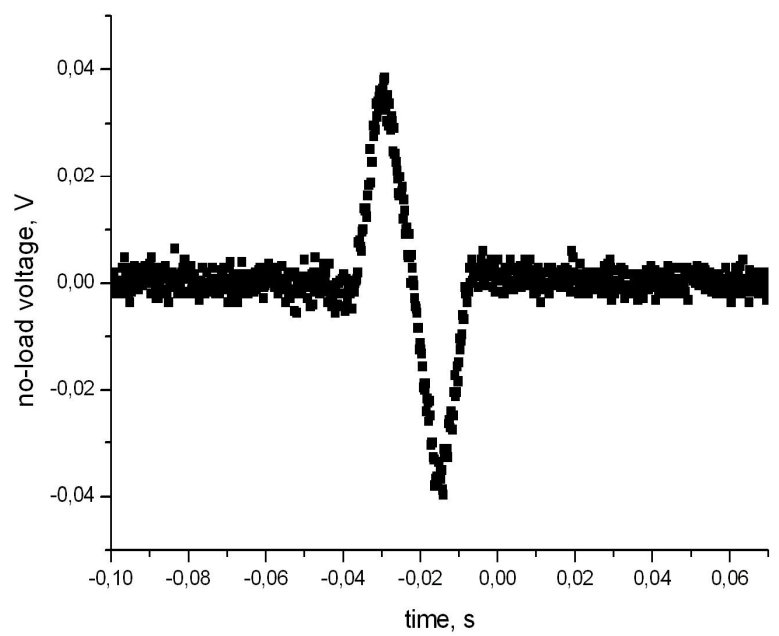

Fig.2. Generated no-load voltage in a coil with 25 turns during one motion along the coil of a block-shaped magnet with dimensions $20 \times 10 \times 5 \mathrm{~mm}$ and remanence 0,1 T.

Generator operation. The alternating magnetic field flux, which goes through the crossection of the FC can be obtained if the axis (south-north direction) of the block-shaped permanent magnet coincides with the direction of the oscillation of the magnet accordingly to FC (Fig.1, line MM'). To achieve a difference from zero, the velocity of the magnetic field changes and during the motion of the magnet its length must be close to (or coincide) with the FC outer diameter. In this case the 
frequency of the voltage generated in the $\mathrm{FC}$ will be equal to the magnet's oscillation frequency (in the case - of one coil).

The change of the flux in time is increased due to the permanent change of the number of turns, which are crossed by a magnetic field, thus, even an equal intensity of a magnetic field creates a different flux in different places of the FC. Figure 2 shows the pulse of no-load voltage, formed in the FC with 25 turns, during a single motion of the magnet along the coil.

Optimization of the generator. Considering the geometry of the FC, it can be concluded that increasing the number of turns would lead to the increase of the coil outer diameter. When integrating the FC into the apparel, an increase of its size is not desirable, since it can change the properties of clothing. Therefore, the possibility of increasing the generated voltage by increasing the number of FCs is being investigated. It is possible by placing the FCs both above one another (with an insulating layer in between) in a way that the magnet in motion moves over them at one time. Fig.3. shows the total value of generated voltage as a function of the FC number (coils were connected into series; the magnetic flux crossed all coils simultaneously).

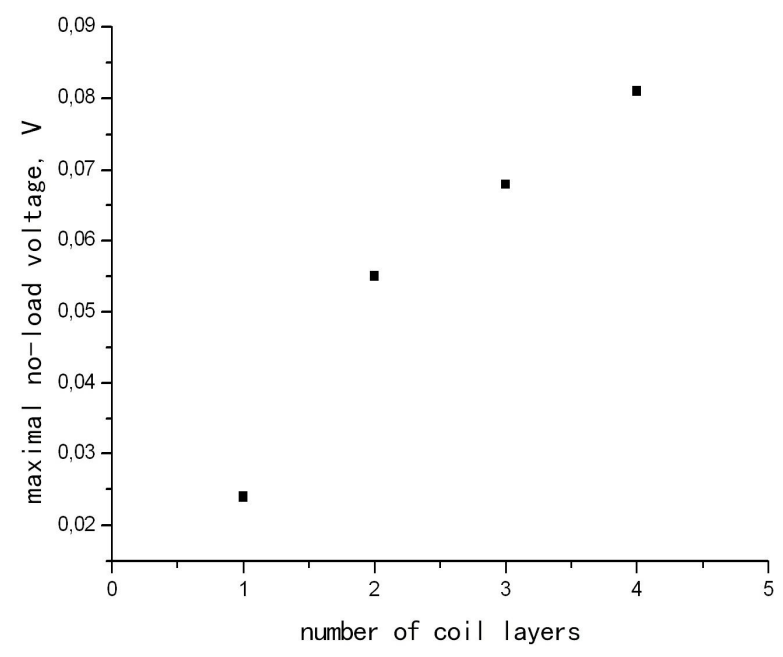

Fig.3. The maximal generated no-load voltage, as function of the number of above one another placed, in a series connected coils. Each coil had 25 turns.

Fig.3. shows that the performance of the generator can be significantly increased by placing multiple, mutually in series connected FCs. The observed indirect dependence of no-load voltage is due to the increased distance between the current layer and the magnet and the shielding activities of upper layers. As restrictive factors for a number of layers the increased weight, thickness and hardness of the apparel with integrated generator can be mentioned. Integrating the multi-coil generator into the apparel, the total layer thickness should not exceed, depending on the type of garment and the integration site, 7 to $10 \mathrm{~mm}$.

As one of the possible places for the deployment of the generator, the outer side of the pocket area (for the FC) and the sleeve (for the magnet) have been defined. The available space for such an arrangement, which remains relatively flat during the motion, is about $10 \times 10 \mathrm{~cm}$. Using a small and lightweight magnet and sticking to the requirements of the similarity of the magnet length and coil diameter, the possibility to place two FCs in a way that a magnet, transits after one another in sequence during a motion, have been studied. In this case, when the magnet is in motion a variable flux of the magnetic field will develop at the beginning in one coil, then - in the second. By changing the distance between the coils we try to pick a position when the induced electromotive forces reinforce each other. This is possible in the case when the magnet simultaneously develops an increasing flux of one pole of the magnet through the second FC, and in the first FC still exists a decreasing flux created by the opposite pole of the magnet. The electromotive forces in two coils will be with similar sigh and will reinforce each other. 
The performance of such a two-coil generator depends also on the distance between the coils, as shown in Fig. 4. It has become clear that all mentioned above reinforcing is most effectively fulfilled in a situation when the distance between the coils is $3 / 4$ of the length of the magnet. The total generated energy in this case is about $4 \mu \mathrm{J}$, the maximum power - $58 \mu \mathrm{W}$ and the maximal no-load voltage - $54 \mathrm{mV}$. Similar energy in two isolated coils is generated at a distance of 1.5 lengths of the magnet, but no-load voltage, which is an important parameter for an energy accumulation system, in this case is $20 \%$ lower.

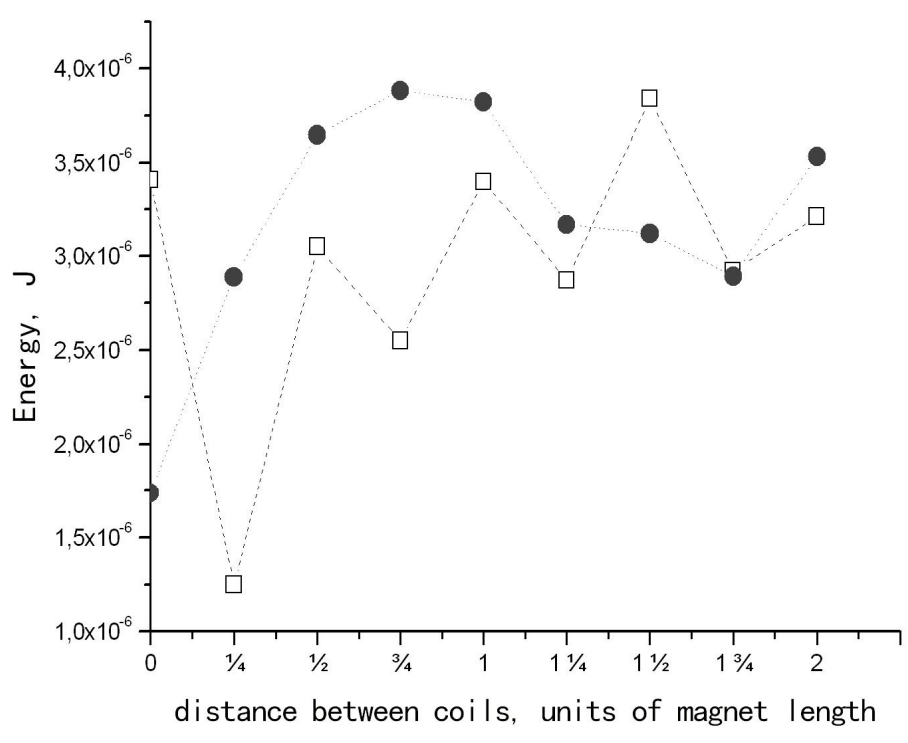

Fig.4. Generated energy as a function of the distance between two coils with 27 turns each: • energy generated in two, sequentially connected coils, $\square$ the total energy generated in two isolated coils.

\section{Discussion}

Such generators can be implemented in virtually all types of clothing, where there are two closely moving parts during the human movement. In this case, the generator frequency will coincide with the frequency of motion of clothing parts with integrated parts of the generator (FC or magnet). It is also possible to implement the inertial or resonant set-up. In this case the moving part of the generator (the magnet for instance) is suspended elastically. The human motion can be used as driven force to induce the resonant oscillations of a magnet. The generators with such resonant structure can also be integrated into garments, as well as accessories (e.g. bags) and can also be used for energy harvesting from other sources with mechanical oscillations.

\section{Conclusion}

The possibility of creating an electrodynamic human movement energy converter with a flat architecture is under investigation. The investigated converter consists of a flat, Archimedes spiralshaped coil and a rectangular magnet. Such an electrodynamic converter can be completely integrated into human apparel and the generated electrical energy can either be stored or used for running various electronic devices.

The possibilities of increasing the developed power of the generator by increasing the number of layers of coils and deploying a two-coil system along the magnet movement path is being investigated. It has been shown that it is possible to increase the total generated power by $52 \%$ compared to the power generated by two insulated coils, by placing two coils connected in series along the magnet movement path, and optimizing the magnetic flux going through them.

\section{References}

[1] Information on: http://ldt.stanford.edu/ jeepark/jeepark+portfolio/cs147hw8jeepark.html

[2] C.R.Saha et al., Sensors and Actuators A vol.147 (2008) pp.248-253.

[3] P.D. Mitcheson et al., Proceedings of the IEEE, vol.96, No. 9, (2008) 1457-1486. 